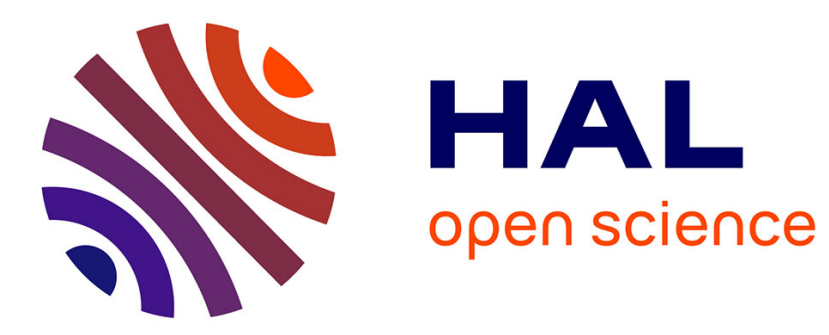

\title{
Demande institutionnelle et responsabilité des chercheurs: langues, insertions, pluralité des parcours et des perceptions
}

\author{
Véronique Castellotti, Emmanuelle Huver, Fabienne Leconte
}

\section{To cite this version:}

Véronique Castellotti, Emmanuelle Huver, Fabienne Leconte. Demande institutionnelle et responsabilité des chercheurs: langues, insertions, pluralité des parcours et des perceptions. De Gruyter Mouton. The linguistic integration of adult migrants: some lessons from research / L'intégration linguistique des migrants adultes : les enseignements de la recherche, , pp.425-431, 2017, 10.1515/9783110477498-058 . hal-01500783

\section{HAL Id: hal-01500783 \\ https://hal.science/hal-01500783}

Submitted on 3 Apr 2017

HAL is a multi-disciplinary open access archive for the deposit and dissemination of scientific research documents, whether they are published or not. The documents may come from teaching and research institutions in France or abroad, or from public or private research centers.
L'archive ouverte pluridisciplinaire $\mathbf{H A L}$, est destinée au dépôt et à la diffusion de documents scientifiques de niveau recherche, publiés ou non, émanant des établissements d'enseignement et de recherche français ou étrangers, des laboratoires publics ou privés. 


\section{Véronique Castellotti, Emmanuelle Huver et Fabienne Leconte Demande institutionnelle et responsabilité des chercheurs : langues, insertions, pluralité des parcours et des perceptions}

Résumé : Cet article vise à questionner la place, le rôle et la responsabilité des chercheurs en sociolinguistique et en didactique des langues dans le domaine de l'intégration linguistique des migrants, domaine de recherche-intervention particulièrement à l'interface de la demande politique et sociale. Pour ce faire, nous interrogerons quelques évidences sur lesquelles se fondent les politiques linguistiques d'intégration et les formations linguistiques qui en résultent. Nous évoquerons notamment la corrélation usuellement établie entre langue et intégration ainsi que les modalités de prise en compte de la diversité linguistique, culturelle, mais aussi biographique dont les migrants sont porteurs.

Abstract: This article sets out to examine the place, role and responsibility of researchers in sociolinguistics and language education with regard to the linguistic integration of migrants, an area of interventional research that is very much at the confluence of political and social demands. To this end the authors will analyse a number of obvious facts on which linguistic policies for integration and the resulting language courses are based. In particular, this article will address the correlation usually established between language and integration, and also ways of taking into account migrants' linguistic, cultural and biographical diversity.

\section{Introduction}

La question des rapports entre recherche, politique et intervention est désormais ancienne et classique (Beacco 2013). Les sociolinguistes et les didacticiens des langues sont actuellement particulièrement concernés, du fait de la place croissante (et renouvelée) accordée aux dimensions linguistiques et culturelles

\footnotetext{
Véronique Castellotti, Université F. Rabelais, Tours, France,
} E-Mail : veronique.castellotti@univ-tours.fr

Emmanuelle Huver, Université F. Rabelais, Tours, France, E-Mail : huver@univ-tours.fr Fabienne Leconte, Université de Rouen, France, E-Mail : fabienne.leconte@univ.rouen.fr

DOI 10.1515/9783110477498-058, (cc)BY-NC-ND (c) 2017 Véronique Castellotti, Emmanuelle Huver et Fabienne Leconte, published by De Gruyter.

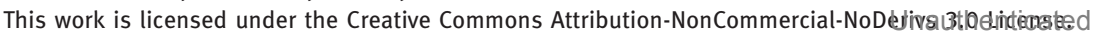


dans les politiques d'accueil et d'insertion des migrants, en Europe plus particulièrement (Bretegnier 2011).

Dans ce domaine, la demande actuellement adressée à la recherche par les instances politiques relève essentiellement de l'application : il s'agit de " tirer profit des enseignements de la recherche » pour « éclairer les décisions » et " aider à élaborer des mesures cohérentes et efficaces » (appel à communication de ce symposium).

Différents éléments poussent les chercheurs à s'aligner (plus ou moins volontairement) sur cette visée pragmatique. On peut (rapidement) relever les éléments suivants :

1. la position d'expertise dans laquelle est ainsi placé le chercheur lui procure un certain nombre d'avantages (financement de ses recherches, visibilité, prestige, etc. $)^{1}$;

2. « l'interaction avec l'environnement social » constitue désormais un important critère d'évaluation des chercheurs eux-mêmes ;

3. un positionnement critique sur la demande est rapidement interprété (y compris par les chercheurs) comme un désengagement politique (voire moral) des chercheurs de la vie de la cité (cf. la métaphore fréquemment convoquée de la tour d'ivoire), a fortiori lorsque les enjeux sont présentés sous l'angle de l'urgence, de l'humanitaire ou du sécuritaire.

Or, cette conception utilitariste de la recherche cantonne le chercheur, au pire à un rôle de caution scientifique, au mieux à un rôle de prestataire au service de l'ingénierie sociale (i.e. de « bonnes pratiques » directement utilisables et reproductibles), contribuant au maintien et - donc - à la diffusion des implicites sur lesquels la demande se fonde.

Il n'est pas question de défendre ici l'idée que la recherche devrait nécessairement se concevoir hors de toute préoccupation ou visée sociopolitique et/ou interventionniste ou d'adopter une attitude moralisante en émettant des jugements a priori sur l'éthique des chercheurs dans des situations de collaboration avec des institutions. Nous considérons au contraire que la sociolinguistique et la didactologie/didactique des langues constituent, justement, des disciplines d'intervention, dans lesquelles les relations entre recherche et intervention sont à envisager comme des relations en tension, la tension elle-même constituant un ferment de la réflexion, à condition qu'elle soit problématisée comme telle.

1 La polémique autour du Français Langue d'Intégration en France illustre en partie cela, même si elle ne se résume pas à des enjeux de pouvoir, un certain nombre d'enjeux sociopolitiques étant également très fortement présents. 
Nous avons choisi d'exemplifier cette réflexion en mobilisant deux problématiques qui sont souvent traitées sur le mode de l'évidence dans les discours et les représentations circulants (y compris au sein de la communauté scientifique elle-même parfois) et qu'il revient donc aux chercheurs de questionner, nuancer, remettre en question : le lien langue(s)/intégration d'une part et la place et le statut de diverses formes de diversité dans les situations de migration d'autre part. Pour ce faire, nous nous focaliserons plus spécifiquement sur le cas de la France, dans la mesure où le lien entre langue, intégration et cohésion nationale y est particulièrement prégnant ${ }^{2}$.

\section{Langue(s) et intégration}

La corrélation entre langue et intégration est souvent pensée comme une « évidence », la maitrise de la langue constituant un " gage » d'intégration réussie (Hambye \& Romainville 2014). Or, si les dimensions linguistiques constituent un atout, ce n'est pas nécessairement ni toujours le plus important, ni un facteur suffisant pour assurer la socialisation (que nous emploierons ici de préférence à intégration), pas plus que des compétences partielles dans la langue du pays d'accueil seraient source principale de difficultés. La langue intervient alors comme un prétexte pour masquer une conception à sens unique de l'intégration, dont une langue et une seule serait le vecteur principal. Pourtant, d'autres facteurs sont souvent largement aussi influents.

\subsection{La socialisation socio-professionnelle}

Dans certains secteurs, l'expérience antérieure du métier et des missions exercées et sa confrontation avec celle d'autres professionnels est primordiale, à la fois dans la socialisation et l'appropriation langagière progressive. En témoignent les propos de médecins étrangers travaillant en France, qui expliquent que leur connaissance de la pathologie s'applique aussi bien ici qu'ailleurs et que le discours n'est pas toujours déterminant dans l'accomplissement du métier. Ils décrivent aussi des formes d'intercompréhension, avec notamment l'anglais ou le latin comme « passerelles » (Levacic 2016). Ce n’est donc pas ici la compétence

2 Cet article est complémentaire de ceux d'E. Lebreton, E. Mercier et A. Bruneau, également publiés dans ce volume et qui illustrent et approfondissent les orientations esquissées dans ce texte. 
langagière qui précède ou conditionne l'insertion professionnelle et la socialisation, mais parfois l'inverse, et la communication professionnelle s'avère alors souvent plus aisée que la communication «familière ». On peut donc, dans de telles situations, imaginer des formations linguistiques qui s'appuient sur la communication professionnelle pour favoriser le développement de compétences plus générales.

\subsection{Pluralité linguistique, réception, appropriation}

En France, les formations sont organisées autour de l'apprentissage exclusif du français, sans mobiliser, voire en interdisant, la présence d'autres expériences langagières. Cette conception, s'appliquant aux migrants comme aux formateurs, est souvent néfaste aux tentatives de socialisation (Mercier ici-même). Il importe donc de réorienter les formations linguistiques vers la pluralité des compétences, y compris en tenant compte du rôle que peuvent jouer des variétés régionales ou dialectales (Eloy 2003).

La dimension prioritairement productive des formations renforce en outre la dimension négative de cet exclusivisme linguistique. En effet, les capacités en réception sont souvent plus élevées que celles en production, qui pourraient alors - partiellement et selon les cas - s'exprimer de façon plurielle. Cela invite à rééquilibrer les formations vers le pôle réceptif de l'apprentissage en valorisant la compréhension et en sensibilisant les accueillants et les formateurs à une tolérance à l'erreur et à l'approximation.

Cela conduit aussi à interroger la notion de " maitrise ", qui entraîne l'idée de perfection, de centration sur le code et les structures, et appelle le contrôle pour vérifier l'exactitude, a-situationnelle. La maitrise, supposée la même pour tous, oriente les formations de façon instrumentale, avec un idéal d'exhaustivité, où le résultat détermine le contenu et l'évaluation pilote l'ensemble du dispositif (Mercier, ici-même). Nous proposons à l'inverse une politique formative reposant sur une exigence d'appropriation, liée à des histoires singulières, qui fasse sens dans des parcours d'expérience diversifiés et qui permette aux personnes migrantes de devenir non seulement acteurs de leur socialisation, mais auteurs de celle-ci. ${ }^{3}$

3 Ce qui, en retour, met à mal la visée de contrôlabilité dont les formations linguistiques actuelles relèvent massivement. 


\section{L'occultation de la diversité langagière et sociale}

Depuis une vingtaine d'années, les migrations se diversifient socialement et géographiquement. Les répertoires langagiers des migrants sont ainsi souvent plus complexes, davantage littéraciés. Aux langues premières et de scolarisation, il faut ajouter des langues de socialisation ou véhiculaires.

La diversité concerne aussi les ressortissants de l'UE : la chute du rideau de fer a encouragé les migrations Est-Ouest. Pourtant, les politiques linguistiques des États ont tendance à occulter les langues parlées avant/pendant la migration et à réduire les politiques publiques à la seule langue du pays d'accueil. Le fait de lier droit au séjour et performance linguistique conduit à différentes catégorisations qui ont peu à voir avec les répertoires langagiers, les qualifications, les projets des personnes. Nous en retiendrons trois : l'appartenance à l'UE, la durée du séjour et la scolarisation.

\subsection{Appartenance à l'Union Européenne}

En France, seuls les non-Européens sont concernés par le Contrat d'Intégration Républicaine, et donc, les formations linguistiques qui en relèvent. L'effet peut être paradoxal : par exemple, dans le cadre d'un Programme Régional d'Intégration des Populations Immigrées, seules les femmes en situation régulière originaires d'un pays tiers ont été retenues pour le programme Accompagnement des femmes migrantes. Les Roumaines ainsi que beaucoup de femmes d'origine maghrébine ayant obtenu la nationalité espagnole ou italienne dans ces pays ont été exclues du dispositif. Elles ressemblaient pourtant au public ciblé.

Se dessine en creux la figure d'un Européen mobile d'un pays à l'autre, qui n'aurait ni de besoins langagiers lors de ses déplacements (il parle « l'européen » ou toutes les langues de l'Union), ni de besoins d'informations spécifiques sur les institutions du pays où il réside désormais. La diversité linguistique, culturelle et biographique, y compris européenne, se trouve ainsi réduite et occultée.

\subsection{Durée du séjour}

Ce même dispositif était réservé aux femmes en France depuis moins de cinq ans. L'État français considère qu'au-delà de 5 ans de présence, les besoins langagiers des migrants ne concernent plus que l'analphabétisme ou l'illettrisme 
(cf. circulaire Valls de mars 2015). Le critère de la durée du séjour est en outre corrélé à l'obtention des cartes de séjour.

Le droit au séjour et à la nationalité française est de plus en plus conditionné par un niveau de langue attesté. La carte de séjour délivrée est d'abord d'un an, à l'issue duquel le niveau Diplôme initial de langue française (DILF) est censé être obtenu. Puis une carte pluriannuelle jusqu'à 4 ans peut être obtenue, un niveau A2 est censé être alors acquis, lequel permettra l'obtention d'une carte de résident. C'est le sens de la loi du 7 mars 2016 qui modifie l'article 2 : « la connaissance suffisante de la langue française » requise pour un titre de séjour devient « connaissance de la langue française qui doit être au moins égale à un niveau défini ». Le dossier de presse accompagnant la loi stipule qu'il s'agit du niveau A2, mais le danger de cette formulation est que le niveau de langue, non inscrit dans la loi, peut augmenter sur simple décret.

\subsection{Scolarisation}

L'organisation des formations linguistiques se fonde sur le critère de la scolarisation : sont identifiées comme relevant du « FLE » les personnes scolarisées. Elles sont alors différenciées des «personnes peu ou pas scolarisées », dénommées « alpha » ou « post-alpha ». Plus récemment, ont été organisées des formations FLI qui préparent les personnes au test linguistique (B1 oral) pour accéder à la nationalité. Comme la passation des tests tend à déterminer le droit au séjour, le risque est grand que cette instrumentalisation des formations se généralise. Dans tous les cas, la complexité et la richesse des répertoires des migrants se trouvent réduites à des manques de compétences, à l'écrit ou en français. Les langues d'appui (anglais, arabe, russe, etc.) sont rarement prises en compte dans les démarches formatives et uniquement là où les formateurs ont été initiés aux démarches plurilingues.

\section{Conclusion}

Lorsque les chercheurs répondent à une demande institutionnelle sans l'interroger, ils contribuent à donner aux préconisations effectuées des allures de pratiques inattaquables, puisqu'elles apparaissent alors comme des dispositifs techniques scientifiquement légitimés. Cependant, toute politique se fonde sur des présupposés qui restent bien souvent implicites, comme l'illustrent les quelques exemples présentés ici. Or, le fait que ces présupposés restent implicites tend à occulter la part de choix (la part de politique, donc) dont ils éma- 
nent. Interroger (publiquement) la demande institutionnelle (et non la critiquer " gratuitement ») constitue ainsi une condition (éthique) de l'intervention de recherche, y compris si la demande se fonde sur des valeurs et des idéaux qui correspondent aux valeurs et idéaux personnels du chercheur.

Il ne suffit pas, pour venir à bout de la problématique, d'ajouter des considérations éthiques aux préconisations techniques effectuées. Il s'agit au contraire d'historiciser et d'épistémologiser la réflexion (et notamment les notions mobilisées), pour que les choix qui fondent la demande puissent être mis en évidence et, ainsi, mis en discussion, la discussion constituant le socle de tout débat démocratique.

\section{Références}

Beacco, Jean-Claude (dir.). 2013. Éthique et politique en didactique des langues. Paris : Didier.

Bretegnier, Aude (dir.). 2011. Formation linguistique en contextes d'insertion. Compétences professionnelles, posture, professionnalité : concevoir un cadre de référence(s). Berne : Peter Lang.

Eloy, Jean-Michel. 2003. Langues d'origine, langues régionales, français. Intégration et plurilinguisme, Ville-École-Intégration Enjeux. 133. 134-146.

Hambye, Philippe \& Anne-Sophie Romainville. 2014. Apprentissage du français et intégration. Des évidences à interroger. Français \& Société. 26-27.

Leconte, Fabienne (dir.). 2016. Adultes migrants, langues et insertions sociales. Dynamiques d'apprentissage et de formation. Paris : Riveneuve.

Levacic, Michelle. 2016. Mobilisation et transformation de compétences professionnelles et langagières dans des parcours de mobilité géographique et culturelle de médecins allophones. Tours : Université F. Rabelais (Tours), thèse de doctorat. 
